\title{
A case of uterine corpus cancer with definitive staging after laparoscopic surgery, and preliminary diagnosis based on endometrial cytology
}

Jiao Shen*, Noriatsu Saeki, Fujihiro Oka, Masayo Onoue, Yuko Yano, Koji Hatta, Takanori Takayama, Ayako Hosoi, Kana Yoshimi, Kimiaki Ozaki, Takao Funato and Namiko Hashimoto

Obstetrics and Gynaecology department, Nippon life hospital, 2-1-54, Enokojima, Nisi-ku, Osaka, Japan

\begin{abstract}
Endometrial cancer should be considered in women with inappropriate vaginal bleeding, menstrual abnormalities, or discolored vaginal discharge. We experienced a case without the above risk factors, in which a preliminary diagnosis was made with endometrial cytology. The case was of a 66-year-old woman, gravida 2 and para 2. She had no inappropriate vaginal bleeding, and transvaginal ultrasound examination showed a thin endometrium. However, endometrial cytology was positive. We performed laparoscopic hysterectomy and bilateral salpingo-oophorectomy based on a clinical diagnosis of Stage IA uterine corpus cancer. The postoperative pathologic diagnosis was stage IB Grade 2 endometrial adenocarcinoma. Therefore, the possibility of uterine corpus cancer must still be considered in cases without risk factors. And cytologic sampling should be considered an effective method for diagnosis of uterine corpus cancer because of its high sensitivity and specificity.
\end{abstract}

\section{Introduction}

Uterine corpus cancer is generally suspected when inappropriate vaginal bleeding occurs and/or a thickened endometrium is detected with transvaginal ultrasound or pelvic magnetic resonance imaging (MRI). However, uterine corpus cancer sometimes occurs without typical signs and symptoms. We experienced a case without any risk factors, in which a preliminary diagnosis was made with endometrial cytology. Staging was confirmed after laparoscopic surgery.

\section{Case report}

A 66-year-old, gravida 2, para 2 patient was menopausal at the age of 46 . She denied any endocrine disorders or lifestyle-related disease factors, such as diabetes or hypertension. She had no inappropriate vaginal bleeding, and transvaginal ultrasound examination showed a thin endometrium. However, endometrial cytology was positive (Figure 1a-b). Dilation and curettage was performed, but only a few adenocarcinoma cells were identified, making definitive diagnosis impossible (Figure 1c-e).

MRI did not provide additional information because of the thin endometrium and internal junctional zone. Contrast computed tomography did not reveal abnormal findings (Figure 2).

We performed laparoscopic hysterectomy and bilateral salpingooophorectomy based on a clinical diagnosis of Stage IA uterine corpus cancer. Although recommended for accurate staging, lymph node dissection was not performed. Only rapid cytodiagnosis of abdominal ascites fluid was performed, and greater omentum segmental resection was omitted because of a negative result (Figure 3).

The postoperative pathologic diagnosis was stage IB Grade 2 endometrial adenocarcinoma (Figure 4). Chemotherapy was performed for moderate risk of recurrence. However, there was no evidence of recurrence at 6 months postoperatively.

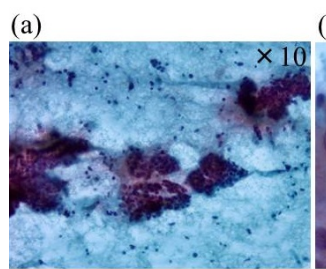

(b)

(c)

(d)
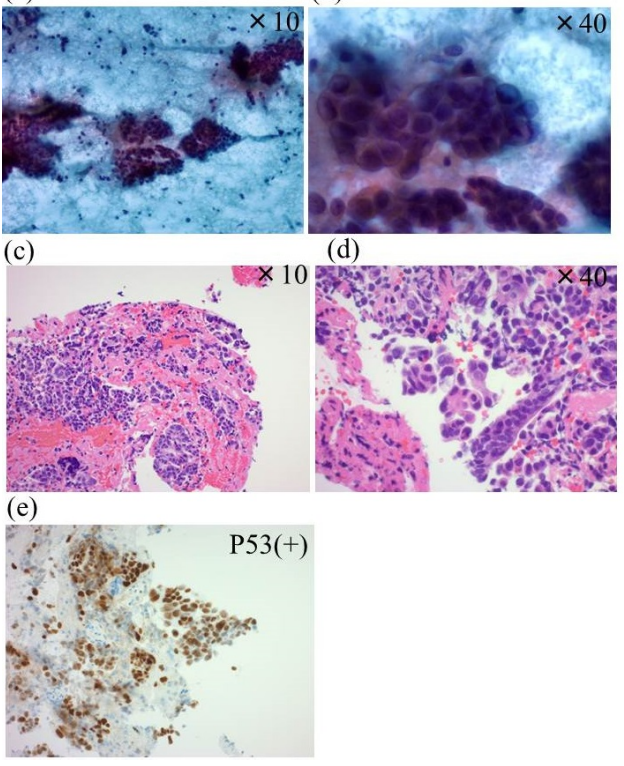

$53(+)$

Figure 1. Preoperative pathological findings. (a-b) Endometrial cytology. Small quantities of atypical acinar cells in a relatively uniform background, consistent with adenocarcinoma. (c-e) A few adenocarcinoma cells seen. The type and grade are unknown; no papillary structures were identified. Diffuse P53 expression was observed (e).

${ }^{\star}$ Correspondence to: Jiao Shen, Obstetrics and Gynaecology department, Nippon life hospital, 2-1-54, Enokojima, Nisi-ku, Osaka, Japan, 550-0006, Tel: 06-6443-3446; E-mail: shenyang51@hotmail.com

Key words: corpus cancer, endometrial cytology, vaginal bleeding

Received: September 05, 2018; Accepted: September 14, 2018; Published: September 17, 2018 

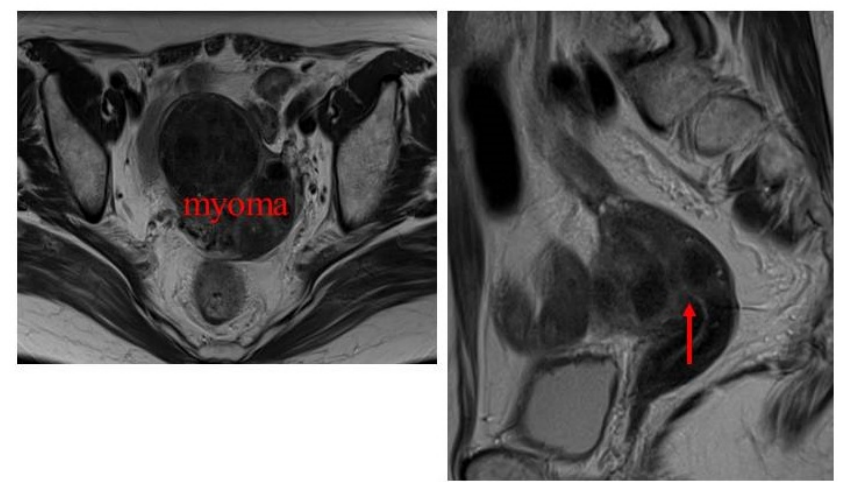

Figure 2. Pelvic magnetic resonance imaging (MRI). Axial and sagittal images show that the endometrium is thin and junction zone is internal (a)

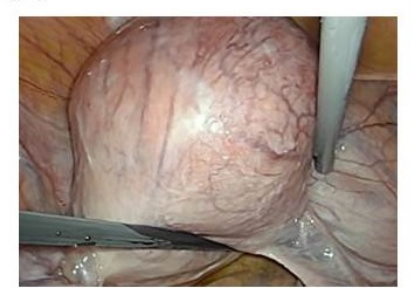

(c)

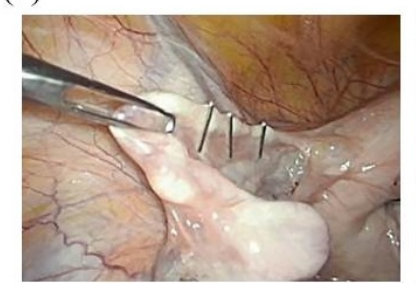

(b)

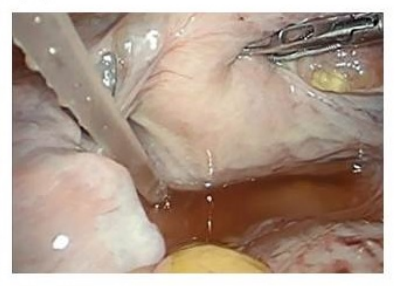

(d)

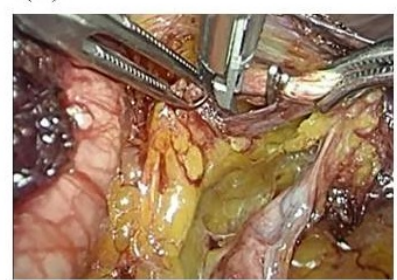

Figure 3. Intraoperative findings. The uterus showed myomatous enlargement (a). No macroscopic dissemination was apparent. Abdominal fluid was collected for rapid cytodiagnosis (b). The Fallopian tubes were ligated, the ureters and uterine arteries were identified (c-d), the bilateral adnexa were examined, the vaginal cuff was incised, and the uterus was removed (a)

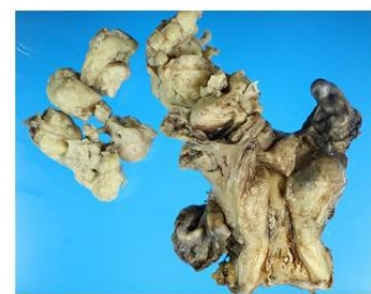

(c)

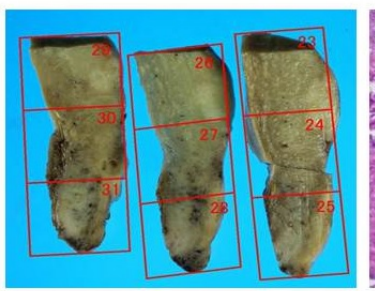

(b)

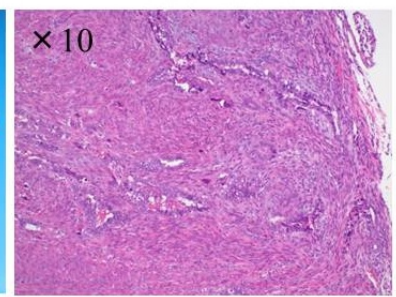

(d)

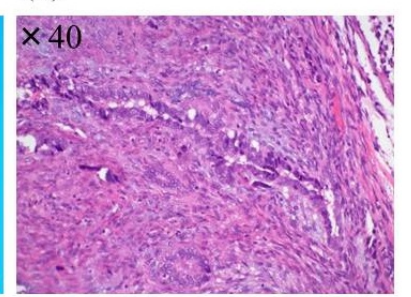

Figure 4. An atypical epithelial lining with dyskaryosis formed glandular structures; the tissue was invasive and hyperplastic. Neoplastic cells deeply infiltrated the myometrium. No direct serosal exposure or vessel penetration was apparent. There was no evidence of cervical cancer

\section{Discussion}

Endometrial cancer should be considered in women with inappropriate vaginal bleeding, menstrual abnormalities, or discolored vaginal discharge. Inappropriate vaginal bleeding is reported in $90 \%$ of endometrial cancer cases, leading to the initial work-up [1].

The sensitivity for a 4-mm cut-off of endometrial thickness measured by transvaginal ultrasound is as high as $91-100 \%$ in a symptomatic postmenopausal woman [2]. Cancer can be ruled out if endometrial thickness is less than $5 \mathrm{~mm} \mathrm{[3].}$

This case showed no abnormal vaginal bleeding or endometrial thickening. Therefore, the possibility of uterine corpus cancer must still be considered in cases without risk factors. Endometrial cytology led to an initial diagnosis of uterine corpus cancer in this case. Fujiwara et.al reported a sensitivity and specificity of $88.5 \%$ and $98.5 \%$, respectively, for endometrial cytodiagnosis, regardless of menstrual status and histologic type [2]. Therefore, cytologic sampling should be considered an effective method for diagnosis of uterine corpus cancer because of its high sensitivity and specificity.

\section{References}

1. Sato S, Chiba Y, Itamochi H, Sugiyama T (2016) Uterine corpus cancer. Japanese J Med Pharm Sci 73: 385-396.

2. Fujiwara H, Takahashi Y, Takano M, Miyamoto M, Nakamura K, et al. (2015) Elevation of endometrial cytology: cytohistological correlation in 1441 cancer patients. Oncology 88: 86-95.

3. Blumenfeld ML, Turner LP (1996) Role of transvaginal sonography in the evaluation of endometrial hyperplasia and cancer. Clin Obstet Gynecol 39: 641-655.
Copyright: (C)2018 Shen J. This is an open-access article distributed under the terms of the Creative Commons Attribution License, which permits unrestricted use, distribution, and reproduction in any medium, provided the original author and source are credited. 